\title{
SHARP GROWTH ESTIMATES FOR WARPING FUNCTIONS IN MULTIPLY WARPED PRODUCT MANIFOLDS
}

\author{
BANG-YEN CHEN AND SHIHSHU WALTER WEI*
}

\begin{abstract}
By applying an average method in PDE, we obtain a dichotomy between "constancy" and "infinity" of the warping functions on complete noncompact Riemannian manifolds for an appropriate isometric immersion of a multiply warped product manifold $N_{1} \times f_{2} N_{2} \times$ $\cdots \times_{f_{k}} N_{k}$ into a Riemannian manifold.

Generalizing the earlier work of the authors in [9], we establish sharp inequalities between the mean curvature of the immersion and the sectional curvatures of the ambient manifold under the influence of quantities of a purely analytic nature (the growth of the warping functions). Several applications of our growth estimates are also presented.
\end{abstract}

MSC: 31B05; 53C21; 53C42.

Keywords: Growth estimate, $L^{q}$ function, warping function, inequality, warped product, minimal immersion, squared mean curvature.

\section{Introduction}

Warped products play very important roles in Differential Geometry and Physics. Examples of warped product include Riemannian manifolds of constant curvature and the best relativistic model of the Schwarzschild spacetime that describes the out space around a mass star or a black hole.

In 9, B.-Y. Chen and S. W. Wei obtained the following necessary condition for an arbitrary isometric immersion of a warped product $N_{1} \times_{f} N_{2}$ into a Riemannian $m$-manifold $\tilde{M}_{c}^{m}$ with sectional curvatures bounded from above by a constant $c$, generalizing the work of B.-Y. Chen in [3] on warped product submanifolds in a Riemannian manifold $R^{m}(c)$ of constant sectional curvature $c$.

Theorem A. 9, Theorem 3.1] For any isometric immersion $\phi: N_{1} \times_{f} N_{2} \rightarrow$ $\tilde{M}_{c}^{m}$ from a warped product $N_{1} \times_{f} N_{2}$ into a Riemannian m-manifold $\tilde{M}_{c}^{m}$ with sectional curvatures bounded from above by a constant $c$, the warping function $f$ satisfies

$$
-\frac{\left(n_{1}+n_{2}\right)^{2}}{4 n_{2}} H^{2}-n_{1} c \leq \frac{\Delta f}{f}
$$

\footnotetext{
*The second author is supported in part by NSF (DMS-1447008).
} 
where $n_{1}=\operatorname{dim} N_{1}$ and $n_{2}=\operatorname{dim} N_{2}, H^{2}=\langle H, H\rangle$ is the squared mean curvature of $\phi$, and $\Delta f$ is the Laplacian of $f$ on $N_{1}$ (defined as the divergence of the gradient vector field of $f, c f$. (2.4)).

The equality sign (1.1) holds if and only if $\phi$ is a mixed totally geodesic immersion with trace $h_{1}=$ trace $h_{2}$, where $h_{1}$ and $h_{2}$ are the restriction of the second fundamental form $h$ of $\phi$ restricted to $N_{1}$ and $N_{2}$, respectively, and at each point $p=\left(p_{1}, p_{2}\right) \in N$, c satisfies $c=K(u, v)=\max K(p)$, for every unit vector $u \in T_{p_{1}}^{1} N_{1}$ and every unit vector $v \in T_{p_{2}}^{1}\left(N_{2}\right)$.

On the other hand, the second author extended in [15] the scope of $L^{q}$ or $q$-integrable functions on complete noncompact Riemannian manifolds to functions with " $p$-balanced" growth depending on $q$, and introduced the concepts of their counter-part to " $p$-imbalanced" growth (cf. Definition 2.1). By coupling these growth estimates with the above inequality (1.1), Chen and Wei establish in 9] some sharp inequalities between quantities of a geometric nature (the mean curvature of the immersion, the sectional curvatures of the ambient manifold) and quantities of a purely analytic nature (the growth of the warping function).

Theorem B. [9] If $f$ is nonconstant and 2-balanced for some $q>1$, then for every Riemannian $n_{2}$-manifold $N_{2}$ and every isometric immersion $\phi$ of the warped product $N_{1} \times_{f} N_{2}$ into any Riemannian manifold $\tilde{M}_{c}^{m}$ with $c \leq 0$, the mean curvature $H$ of $\phi$ satisfies

$$
H^{2}>\frac{4 n_{1} n_{2}|c|}{\left(n_{1}+n_{2}\right)^{2}}
$$

at some points.

Hence we immediately find a dichotomy between "constancy" and "infinity" (2-imbalanced) of the warping functions on complete noncompact Riemannian manifolds for an appropriate isometric immersion:

Corollary A. 9] Suppose the squared mean curvature of the isometric immersion $\phi: N_{1} \times_{f} N_{2} \rightarrow \tilde{M}_{c}^{m}$ satisfies

$$
H^{2} \leq \frac{4 n_{1} n_{2}|c|}{\left(n_{1}+n_{2}\right)^{2}}
$$

everywhere on $N_{1} \times_{f} N_{2}$. Then the warping function $f$ is either a constant or it has 2-imbalanced growth for every $q>1$.

Applications of these new inequalities are also presented, among which there are some results on the nonexistence of isometric minimal immersions between certain types of Riemannian manifolds:

Theorem C. 9] Suppose $q>1$ and the warping function $f$ is 2-balanced. If $\mathrm{N}_{2}$ is compact, then there does not exists an isometric minimal immersion from $N_{1} \times_{f} N_{2}$ into any Euclidean space. 
A Riemannian manifold is said to be negatively curved (resp., non-positively curved) if it has negative (resp., non-positively curved) sectional curvatures.

Corollary B. 9] If $f$ is an $L^{q}$ function on $N_{1}$ for some $q>1$, then for any Riemannian manifold $N_{2}$ the warped product $N_{1} \times_{f} N_{2}$ does not admit any isometric minimal immersion into any non-positively curved Riemannian manifold.

For further extension, let $N=N_{1} \times \cdots \times N_{k}$ denote the Cartesian product of $k$ Riemannian manifolds $\left(N_{1}, g_{1}\right) \cdots,\left(N_{k}, g_{k}\right)$, and $\pi_{i}: N \rightarrow N_{i}$ be the canonical projection of $N$ onto $N_{i}, 1 \leq i \leq k$. If $f_{2}, \cdots f_{k}: N_{1} \rightarrow \mathbb{R}^{+}$are smooth positive-valued functions, then

$$
g=\pi_{1}^{*} g_{1}+\sum_{i=2}^{k}\left(f_{i} \circ \pi_{1}\right)^{2} \pi_{i}^{*} g_{i}
$$

defines a Riemannian metric on $N$, called multiply warped product metric. The product manifold $N$ endowed with $g$ is denoted by $N=N_{1} \times{ }_{f_{2}} N_{2} \times$ $\cdots \times_{f_{k}} N_{k}$.

Denote by trace $h_{i}$ the trace of the second fundamental form $h$ of $N=$ $N_{1} \times \cdots \times N_{k}$ into a Riemannian manifold restricted to $N_{i}$.

B.-Y. Chen and F. Dillen proved in [6] the following.

Theorem D. Let $\phi: N_{1} \times f_{2} N_{2} \times \cdots \times{ }_{f_{k}} N_{k} \rightarrow M$ be an isometric immersion of a multiply warped product $N=N_{1} \times f_{2} N_{2} \times \cdots \times f_{k} N_{k}$ into an arbitrary Riemannian manifold $M$. Then we have

$$
-\frac{n^{2}(k-1)}{2 k} H^{2}-n_{1}\left(n-n_{1}\right) \max \tilde{K} \leq \sum_{j=2}^{k} n_{j} \frac{\Delta f_{j}}{f_{j}},
$$

where $n=\sum_{i=1}^{k} n_{i}$ and $\max \tilde{K}(p)$ denotes the maximum of the sectional curvature function of the ambient space $M$ restricted to 2-plane sections of the tangent space $T_{p} N$ of $N$ at $p=\left(p_{1}, \ldots, p_{k}\right)$.

The equality sign of (1.4) holds identically if and only if the following two conditions hold

i) $\phi$ is mixed totally geodesic such that trace $h_{1}=\cdots=$ trace $h_{k}$;

ii) At each point $p \in N$, the sectional curvature function $\tilde{K}$ satisfies $\tilde{K}(u, v)=\max \tilde{K}(p)$ for every $u \in T_{p_{1}}^{1} N_{1}$ and every $v \in T_{p_{2}, \ldots p_{k}}^{1}\left(N_{2} \times\right.$ $\left.\cdots N_{k}\right)$.

One main purpose of this article is to prove the following theorem which extends Theorem B, in particular, inequality (1.2) to arbitrary isometric immersions of multiply warped product manifolds into an arbitrary Riemannian manifold.

Theorem 1.1. If for each $j, 2 \leq j \leq k, f_{j}$ is nonconstant and 2-balanced with some $q_{j}>1$, then, for any multiply warped product $N=N_{1} \times_{f_{2}} N_{2} \times$ 
$\cdots \times{ }_{f_{k}} N_{k}$ in a Riemannian manifold $M$, the mean curvature $H$ of $N$ in $M$ satisfies

$$
H^{2}>\frac{-2 k n_{1}\left(n-n_{1}\right)}{n^{2}(k-1)} \max \tilde{K}
$$

at some points, where $\max \tilde{K}$ is defined in Theorem $D$.

In particular, if each $f_{j}$ is nonconstant and in $L^{q_{j}}$ for some $q_{j}>1$, then (1.5) holds at some points.

In particular, if $M$ is a Riemannian manifold of constant sectional curvature $c \leq 0$, then Theorem 1.1 reduces to the following.

Theorem 1.2. If for each $j, 2 \leq j \leq k, f_{j}$ is nonconstant and 2-balanced with some $q_{j}>1$, then, for any multiply warped product $N=N_{1} \times f_{2} N_{2} \times$ $\cdots \times{ }_{f_{k}} N_{k}$ in a Riemannian manifold $R^{m}(c)$ of constant sectional curvature $c \leq 0$, the mean curvature $H$ of $N$ in $R^{m}(c)$ satisfies

$$
H^{2}>\frac{2 k n_{1}\left(n-n_{1}\right)}{n^{2}(k-1)} c
$$

at some points.

In particular, if each $f_{j}$ is nonconstant and in $L^{q_{j}}$ for some $q_{j}>1$, then (1.6) holds at some points.

Theorems 1.1 and 1.2 are sharp and inequalities (1.5) and (1.6) are optimal. For details, we refer to Remark 3.1, Example 3.1, Example 3.2, and Remark 3.2.

In views of Theorem 1.1, we give the following dichotomy.

Theorem 1.3. Suppose the squared mean curvature of the isometric immersion of a multiply warped product $N=N_{1} \times_{f_{2}} N_{2} \times \cdots \times \times_{f_{k}} N_{k}$ into a Riemannian manifold satisfies

$$
H^{2} \leq \frac{-2 k n_{1}\left(n-n_{1}\right)}{n^{2}(k-1)} \max \tilde{K}
$$

everywhere on $N$. Then there exists an integer $i, 2 \leq i \leq k$ such that either the warping function $f_{i}$ is a constant or $f_{i}$ has 2-imbalanced growth for every $q_{i}>1$.

Some other applications of Theorem 1.1 are the following.

Corollary 1.1. If for each $j, 2 \leq j \leq k, f_{j}$ is nonconstant and 2-balanced for some $q_{j}>1$, then there does not exist a minimal immersion of any multiply warped product $N=N_{1} \times_{f_{2}} N_{2} \times \cdots \times{ }_{f_{k}} N_{k}$ into a Riemannian manifold whose maximum sectional curvature is nonpositive.

In particular, if each $f_{j}$ is nonconstant and in $L^{q_{j}}$ for some $q_{j}>1$, then there does not exist a minimal immersion of any multiply warped product $N=N_{1} \times f_{2} N_{2} \times \cdots \times{ }_{f_{k}} N_{k}$ into a Euclidean space. 
Applying the growth estimates in Theorem 2.1 and the average method in PDE in Proposition 2.1, we have the following Liouville property and characterization results.

Corollary 1.2. Suppose the squared mean curvature of the isometric immersion $\phi$ of a multiply warped product $N=N_{1} \times f_{f_{2}} N_{2} \times \cdots \times{ }_{f_{k}} N_{k}$ into a complete, simply-connected Riemannian manifold $R^{m}(c)$ of constant sectional curvature $c$ satisfies (1.7) everywhere on $N$. If for each $j, 2 \leq j \leq k$, $f_{j}$ is 2-balanced for some $q_{j}>1$, then we have:

(1) Every warping function $f_{j}, 2 \leq j \leq k$ is constant.

(2) The isometric immersion $\phi$ is a minimal immersion into a Euclidean space.

(3) The isometric immersion $\phi$ is a warped product immersion.

Corollary 1.3. Let each $f_{j}, 2 \leq j \leq k$ be 2-balanced for some $q_{j}>1$. Then we have:

(1) Every multiply warped product $N=N_{1} \times_{f_{2}} N_{2} \times \cdots \times{ }_{f_{k}} N_{k}$ does not admit an isometrically minimal immersion into any Riemannian manifold of negative sectional curvature.

(2) If $N_{k}$ is compact, then $N=N_{1} \times_{f_{2}} N_{2} \times \cdots \times{ }_{f_{k}} N_{k}$ does not admit an isometrically minimal immersion into a Euclidean space.

We state a special case of Corollary 1.3 as the following.

Corollary 1.4. If each $f_{j}, 2 \leq j \leq k$, is in $L^{q_{j}}$ for some $q_{j}>1$, then we have:

(1) Every multiply warped product $N=N_{1} \times_{f_{2}} N_{2} \times \cdots \times{ }_{f_{k}} N_{k}$ does not admit an isometrically minimal immersion into any negatively curved Riemannian manifold.

(2) If $N_{k}$ is compact, then $N=N_{1} \times_{f_{2}} N_{2} \times \cdots \times{ }_{f_{k}} N_{k}$ does not admit an isometrically minimal immersion into a Euclidean space.

A map

$$
\psi: N_{1} \times_{f_{2}} N_{2} \times \cdots \times_{f_{k}} N_{k} \rightarrow M_{1} \times_{\rho_{2}} M_{2} \times \cdots \times{ }_{\rho_{k}} M_{k}
$$

between two multiply warped product manifolds $N_{1} \times f_{f_{2}} N_{2} \times \cdots \times{ }_{f_{k}} N_{k}$ and $M_{1} \times{ }_{\psi_{2}} M_{2} \times \cdots \times \times_{\psi_{k}} M_{k}$ is said to be a warped product immersion if $\psi$ is given by $\psi\left(x_{1}, \cdots, x_{k}\right)=\left(\psi_{1}\left(x_{1}\right), \cdots, \psi_{k}\left(x_{k}\right)\right)$ is an isometric immersion, where $\psi_{i}: N_{i} \rightarrow M_{i}, i=2, \cdots, k$ are isometric immersions, and $f_{i}=\rho_{i} \circ \psi_{1}$ : $N_{1} \rightarrow \mathbb{R}^{+}$for $i=2, \cdots, k$.

By applying Theorem D, Proposition 2.1 and Theorem E (Nölker's Theorem), we have

Corollary 1.5. If for each $j, 2 \leq j \leq k, f_{j}$ is 2-balanced for some $q_{j}>1$, then every isometric minimal immersion of a multiply warped product $N=$ $N_{1} \times f_{2} N_{2} \times \cdots \times{ }_{f_{k}} N_{k}$ into a Euclidean space is a warped product immersion. 
The technique used in this article is to apply the Average Method in PDE in Proposition 2.1 and the Growth Estimates in Theorem 2.1 to study multiply warped products. In contrast to an extrinsic average variational method in the calculus of variations [16, 17, 10, where the sum of analytic quantities is strictly negative, the average method in PDE in this article deals with the nonnegative sum of analytic quantities (cf. Remark 2.1).

The techniques used in this article are sufficient general to apply to multiply warped product manifolds totally real isometrically immersed into complex space forms, as well as into quaternionic space forms. We also use the same technique for multiply warped product manifolds to treat doubly warped product manifolds in the last section.

\section{Preliminaries.}

Let $N$ be a Riemannian $n$-manifold isometrically immersed in a Riemannian $m$-manifold $\tilde{M}^{m}$. We choose a local field of orthonormal frame $e_{1}, \ldots, e_{n}, e_{n+1}, \ldots, e_{m}$ in $\tilde{M}^{m}$ such that, restricted to $N$, the vectors $e_{1}, \ldots, e_{n}$ are tangent to $N$ and $e_{n+1}, \ldots, e_{m}$ are normal to $N$.

For a submanifold $N$ in $\tilde{M}^{m}$, let $\nabla$ and $\tilde{\nabla}$ denote the Levi-Civita connections of $N$ and $\tilde{M}^{m}$, respectively. The Gauss and Weingarten formulas are then given respectively by (see, for instance, [4, 5])

$$
\begin{aligned}
& \tilde{\nabla}_{X} Y=\nabla_{X} Y+h(X, Y), \\
& \tilde{\nabla}_{X} \xi=-A_{\xi} X+D_{X} \xi
\end{aligned}
$$

for vector fields $X, Y$ tangent to $N$ and $\xi$ normal to $N$, where $h$ is the second fundamental form, $D$ the normal connection, and $A$ the shape operator of the submanifold. Let $\left\{h_{i j}^{r}\right\}, i, j=1, \ldots, n ; r=n+1, \ldots, m$, denote the coefficients of the second fundamental form $h$ with respect to $e_{1}, \ldots, e_{n}, e_{n+1}, \ldots, e_{m}$.

The mean curvature vector $\vec{H}$ is defined by

$$
\vec{H}=\frac{1}{n} \operatorname{trace} h=\frac{1}{n} \sum_{i=1}^{n} h\left(e_{i}, e_{i}\right),
$$

where $\left\{e_{1}, \ldots, e_{n}\right\}$ is a local orthonormal frame of the tangent bundle $T N$ of $N$. The squared mean curvature is given by

$$
H^{2}=\langle\vec{H}, \vec{H}\rangle,
$$

where $\langle$,$\rangle denotes the inner product. A submanifold N$ is called minimal in $\tilde{M}^{m}$ if its mean curvature vector vanishes identically.

Let $P$ be a Riemannian $k$-manifold and $\left\{e_{1}, \ldots, e_{k}\right\}$ be an orthonormal frame field on $P$. For a differentiable function $\varphi$ on $P$, the Laplacian of $\varphi$ is defined by the divergence of the gradient of $\varphi$, or the trace of the Hessian 
$\varphi$, i.e.

$$
\Delta \varphi=\sum_{j=1}^{k}\left\{e_{j} e_{j} \varphi-\left(\nabla_{e_{j}} e_{j}\right) \varphi\right\} .
$$

A function $\varphi$ on $P$ is said to be harmonic (resp. subharmonic or superharmonic) if we have $\Delta \varphi=0$ (resp. $\Delta \varphi \geq 0$ or $\Delta \varphi \leq 0$ ) on $P$.

An isometric immersion

$$
\phi: N_{1} \times_{f_{2}} N_{2} \times \cdots \times{ }_{f_{k}} N_{k} \rightarrow M
$$

of a multiply warped product $N_{1} \times{ }_{f_{2}} N_{2} \times \cdots \times{ }_{f_{k}} N_{k}$ into a Riemannian $m$ manifold $M$ is called mixed totally geodesic if its second fundamental form $h$ satisfies $h\left(\mathcal{D}_{i}, \mathcal{D}_{j}\right)=0$ for any distinct $i, j \in\{1, \cdots, k\}$, where $\mathcal{D}_{i}$ denotes the distribution obtained from the vectors tangent to the horizontal lifts of $N_{i}$.

We recall the following results for later use.

Theorem E. 13, Nölker's Theorem] Let $\phi: N_{1} \times_{f_{2}} N_{2} \times \cdots \times \times_{f_{k}} N_{k} \rightarrow R^{m}(c)$ be an isometric immersion into a Riemannian manifold $R^{m}(c)$ of constant sectional curvature $c$. If $\phi$ is mixed totally geodesic, then locally $\phi$ is a warped product immersion

In the following, let us assume that $N_{1}$ is a noncompact complete Riemannian manifold and $B\left(x_{0} ; r\right)$ denotes the geodesic ball of radius $r$ centered at $x_{0} \in N_{1}$.

We recall some notions from [15].

Definition 2.1. A function on $N_{1}$ is said to have $p$-balanced growth (or, simply, is $p$-balanced) if it is one of the following: $p$-finite, $p$-mild, $p$-obtuse, $p$ moderate, and $p$-small; it has $p$-imbalanced growth, or simply is $p$-imbalanced otherwise.

Notice that the definitions of " $p$-finite, $p$-mild, $p$-obtuse, $p$-moderate, $p$ small" and their counter-parts " $p$-infinite, $p$-severe, $p$-acute, $p$-immoderate, $p$-large" growth depend on $q$, and $q$ will be specified in the context in which the definition is used.

We have discussed their definitions in [9, Definition 4.1-4.5]. For completeness we include them as follows (please see also [15]).

Definition 2.2. A function $f$ on $N_{1}$ is said to have $p$-finite growth (or, simply, is $p$-finite) if there exists $x_{0} \in N_{1}$ such that

$$
\lim _{r \rightarrow \infty} \inf \frac{1}{r^{p}} \int_{B\left(x_{0} ; r\right)}|f|^{q} d v<\infty ;
$$

it has p-infinite growth (or, simply, is p-infinite) otherwise.

Definition 2.3. A function $f$ has $p$-mild growth (or, simply, is $p$-mild) if there exists $x_{0} \in N_{1}$, and a strictly increasing sequence of $\left\{r_{j}\right\}_{0}^{\infty}$ going to 
infinity, such that for every $l_{0}>0$, we have

$$
\sum_{j=\ell_{0}}^{\infty}\left(\frac{\left(r_{j+1}-r_{j}\right)^{p}}{\int_{B\left(x_{0} ; r_{j+1}\right) \backslash B\left(x_{0} ; r_{j}\right)}|f|^{q} d v}\right)^{\frac{1}{p-1}}=\infty ;
$$

and has $p$-severe growth (or, simply, is $p$-severe) otherwise.

Definition 2.4. A function $f$ has p-obtuse growth (or, simply, is p-obtuse) if there exists $x_{0} \in N_{1}$ such that for every $a>0$, we have

$$
\int_{a}^{\infty}\left(\frac{1}{\int_{\partial B\left(x_{0} ; r\right)}|f|^{q} d v}\right)^{\frac{1}{p-1}} d r=\infty
$$

and has p-acute growth (or, simply, is p-acute) otherwise.

Definition 2.5. A function $f$ has $p$-moderate growth (or, simply, is $p$ moderate) if there exist $x_{0} \in N_{1}$, and $F(r) \in \mathcal{F}$,such that

$$
\lim \sup _{r \rightarrow \infty} \frac{1}{r^{p} F^{p-1}(r)} \int_{B\left(x_{0} ; r\right)}|f|^{q} d v<\infty .
$$

And it has p-immoderate growth (or, simply, is p-immoderate) otherwise, where

$$
\mathcal{F}=\left\{F:[a, \infty) \longrightarrow(0, \infty) \mid \int_{a}^{\infty} \frac{d r}{r F(r)}=+\infty \text { for some } a \geq 0\right\}
$$

(Notice that the functions in $\mathcal{F}$ are not necessarily monotone.)

Definition 2.6. A function $f$ has $p$-small growth (or, simply, is p-small) if there exists $x_{0} \in N_{1}$, such that for every $a>0$, we have

$$
\int_{a}^{\infty}\left(\frac{r}{\int_{B\left(x_{0} ; r\right)}|f|^{q} d v}\right)^{\frac{1}{p-1}} d r=\infty
$$

and has p-large growth (or, simply, is p-large) otherwise.

We recall the following result from [9] for later use.

Theorem 2.1 (Warping Function Growth Estimates). Let $N_{1}$ be a noncompact complete Riemannian manifold and $f: N_{1} \rightarrow \mathbb{R}^{+}$be a $C^{2}$ positive function satisfying $\Delta f / f \geq 0$ on $N_{1}$. Then either $f$ is constant or $f$ is 2-imbalanced for every $q>1$.

Proof. Follow exactly the proof of Theorems 4.1, 4.2, 4.3, 4.4 and 4.5 in 9 , p.586-590] and use Definition 2.1, the assertion follows.

We also need the following result.

Proposition 2.1 (An Average Method in PDE). Let $c_{2}, \cdots, c_{k}$ be $k-1$ positive constants and let $f_{2}, \cdots, f_{k}$ be positive-valued functions defined on a complete noncompact manifold $N_{1}$ such that $\sum_{j=2}^{k} c_{j} \Delta f_{j} / f_{j} \geq 0$, Then we have: 
(1) There exists an integer $i, 2 \leq i \leq k$, such that either $f_{i}$ is a constant or $f_{i}$ is 2-imbalanced for every $q_{i}>1$.

(2) If each $f_{j}, 2 \leq j \leq k$, is 2-balanced for some $q_{j}>1$, then all of $f_{2}, \cdots, f_{k}$ are constant functions.

Proof. If $\sum_{j=2}^{k} c_{j} \Delta f_{j} / f_{j} \geq 0$ holds, then there exists at least $i, 2 \leq i \leq$ $k$, such that $\Delta f_{i} / f_{i} \geq 0$ holds. Or $\sum_{j=2}^{k} c_{j} \Delta f_{j} / f_{j}<0$, contradicting to the hypothesis. Therefore statement (1) of this proposition follows from Theorem 2.1.

For statement (2), it follows from the assumptions that $f_{i}$ is 2-balanced. Hence statement (1) implies that $f_{i}$ is constant. So we have $\Delta f_{i}=0$ and

$$
\sum_{j \neq i} c_{j} \frac{\Delta f_{j}}{f_{j}}=\sum_{j=2}^{k} c_{j} \frac{\Delta f_{j}}{f_{j}} \geq 0 .
$$

Now, by applying statement (1) again to $\sum_{j \neq i} c_{j} \Delta f_{j} / f_{j} \geq 0$, we can find the second constant warping function $f_{i^{\prime}}$ such that $\sum_{j \neq i, i^{\prime}} c_{j} \Delta f_{j} / f_{j} \geq 0$. Now, using the same method iteratively, we conclude that all of $f_{2}, \cdots, f_{k}$ are all constant. This proves statement (2).

Remark 2.1. The average method given in Proposition 2.1 is in contrast to an extrinsic average variational method in the calculus of variations [16, 17, 10, where the sum of analytic quantities, the second variation formulas of functionals such as the mass, $p$-energy, or Yang-Mills functional (over a set of distinguished variation vector fields) is strictly negative. Our average method in PDE in Proposition 2.1 deals with the nonnegative sum of analytic quantities, the Laplacian of warping functions.

\section{Proof of Theorem 1.1, Theorem 1.3 and Corollaries 1.1 - 1.5.}

The proofs of these results are based on Theorem D via the Average Method in PDE given in Proposition 2.1 and the Warping Function Growth Estimates given in Theorem 2.1.

Proof of Theorem 1.1. Suppose contrary to (1.5), i.e., there were an isometric immersion $\phi$ whose mean curvature $H$ satisfying

$$
H^{2} \leq \frac{-2 k n_{1}\left(n-n_{1}\right) \max \tilde{K}}{n^{2}(k-1)}
$$

everywhere on $N$. This would imply by multiplying both sides of (1.7) by a positive number $n^{2}(k-1) / 2 k$, or equivalently

$$
0 \leq-\frac{n^{2}(k-1)}{2 k} H^{2}-n_{1}\left(n-n_{1}\right) \max \tilde{K} .
$$

On the other hand, Theorem D would imply

$$
-\frac{n^{2}(k-1)}{2 k} H^{2}-n_{1}\left(n-n_{1}\right) \max \tilde{K} \leq \sum_{j=2}^{k} n_{j} \frac{\Delta f_{j}}{f_{j}} .
$$


After combining this with (3.1) or its equivalent inequality, we find

$$
\sum_{j=2}^{k} n_{j} \frac{\Delta f_{j}}{f_{j}} \geq-\frac{n^{2}(k-1)}{2 k} H^{2}-n_{1}\left(n-n_{1}\right) \max \tilde{K} \geq 0 .
$$

Now, after applying the Average Method in PDE stated in Proposition 2.1(1), we would conclude from (3.2) that some $f_{i}, 2 \leq i \leq k$, could be constant or $f_{i}$ would be 2 -imbalanced for every $q_{i}>1$, contradicting the assumption that $f_{i}$ is nonconstant and 2-balanced for some $q_{i}>1$. Indeed, " $f_{i}$ would be constant" contradicts " $f_{i}$ is nonconstant" and " $f_{i}$ would be 2 imbalanced for every $q_{i}>1$." contradicts " $f_{i}$ is 2 -balanced for some $q_{i}>1$.

To prove the last assertion, we observed that every $L^{q}$ function has 2finite, 2-mild, 2-obtuse, 2-moderate, 2-small growth for the same $q$ (cf. [18, Proposition 2.3]). For example, if $f$ defined on $N_{1}$ is in $L^{q}$, then $f$ is 2-finite with respect to the same $q$. Indeed, there exists $x_{0} \in N_{1}$ such that (2.5), where $p=2$ holds:

$$
\begin{aligned}
\lim _{r \rightarrow \infty} \inf \frac{1}{r^{2}} \int_{B\left(x_{0} ; r\right)}|f|^{q} d v & \leq \lim _{r \rightarrow \infty} \inf \frac{1}{r^{2}} \int_{N_{1}}|f|^{q} d v \\
& =\lim _{r \rightarrow \infty} \inf \frac{1}{r^{2}} C, \text { for some constant } C>0 \\
& =0<\infty .
\end{aligned}
$$

Definition 2.1 and the first assertion of Theorems 1.1 complete the proof.

Proof of Theorem 1.3. Let $\phi: N=N_{1} \times f_{f_{2}} N_{2} \times \cdots \times \times_{f_{k}} N_{k} \rightarrow M$ be an isometric immersion of a multiply warped product $N=N_{1} \times f_{2} N_{2} \times \cdots \times f_{k} N_{k}$ in a Riemannian manifold $M$. If the mean curvature $H$ of $N$ in $M$ satisfies (1.7) on $N$, then it follows from the inequality (1.4) of Theorem D and (1.7) that $\sum_{j=2}^{k} n_{j} \frac{\Delta f_{j}}{f_{j}} \geq 0$. Hence, after applying the Average Method in PDE stated in Proposition 2.1(1), we conclude that some $f_{i}, 2 \leq i \leq k$, could be constant or $f_{i}$ would be 2 -imbalanced.

Proof of Corollary 1.1. Suppose contrary, such an immersion would violate (1.5) and hence contradicts Theorem 1.1.

Proof of Corollary 1.2. Statement (1) of Corollary 1.2 follows from Theorem 1.3 and Proposition 2.1(2).

In view of (1.7) and Proposition 2.1(2), we have

$$
0 \leq-H^{2}-\frac{2 k n_{1}\left(n-n_{1}\right) c}{n^{2}(k-1)} \leq \sum_{j=2}^{k} n_{j} \frac{\Delta f_{j}}{f_{j}}=0 .
$$

Therefore we obtain $H=c=0$, which implies statement (2).

Statement (3) follows immediately from Corollary 1.5. 
Proof of Corollary 1.3. To prove statement (1), let us suppose contrary. Then it follows from Theorem D that

$$
0<-H^{2}-\frac{2 k n_{1}\left(n-n_{1}\right) \max \tilde{K}}{n^{2}(k-1)} \leq \sum_{j=2}^{k} n_{j} \frac{\Delta f_{j}}{f_{j}} .
$$

Now, by Theorem 2.1(1), (3.3) implies the constancy of $f_{i}$ for some $2 \leq i \leq$ $k$. Thus

$$
0<\sum_{j \neq i} \frac{\Delta f_{j}}{f_{j}} .
$$

Therefore, after applying Proposition 2.1(2) to (3.4) we obtain the constancy of $f_{2}, \cdots, f_{k}$, which leads to $0<0$, a contradiction.

For statement (2), let us suppose contrary. Then inequality (3.2) would be true. Hence by Proposition 2.1(1), we would have the constancy of $f_{i}$ for some $2 \leq i \leq k$. Thus

$$
0 \leq \sum_{j \neq i} n_{j} \frac{\Delta f_{j}}{f_{j}}
$$

Now, applying Proposition [2.1(2) shows the constancy of $f_{2}, \cdots, f_{k}$. So, it follows from Theorem $\mathrm{D}$ that $\phi$ is mixed totally geodesic and hence, by Moore's lemma [12, we conclude that

$$
\phi=\left(\phi_{1}, \cdots, \phi_{k}\right): N=N_{1} \times_{f_{2}} N_{2} \times \cdots \times{f_{k}}_{k} N_{k} \rightarrow \mathbb{E}^{m_{1}} \times \cdots \times \mathbb{E}^{m_{k}}=\mathbb{E}^{m}
$$

is a product minimal immersion, which contradicts to the fact that there is no compact minimal submanifold $N_{k}$ in the Euclidean space $\mathbb{E}^{m_{k}}$.

Proof of Corollary 1.4. Follows at once from Corollary 1.3 and the fact that every $L^{q}$ function with $q>1$ on $N_{1}$ is 2-balanced for the same $q>1$ on $N_{1}$.

Proof of Corollary 1.5. In view of Theorem D and $H=c=0$, we have

$$
0=-H^{2}-\frac{2 k n_{1}\left(n-n_{1}\right) c}{n^{2}(k-1)}=\sum_{j=2}^{k} n_{j} \frac{\Delta f_{j}}{f_{j}}=0 .
$$

Now assertion follows from Theorem E [13, Nölker's Theorem].

Remark 3.1. The following two examples show that Theorem 1.1 is false if either $f_{j}$ is constant or $f_{j}$ is 2 -imbalance for every $q_{j}>1$.

Example 3.1. Let $N_{1}, \ldots, N_{k}$ be $k$ copies of the real line $\mathbf{R}$ and let us put $f_{j}=1$ for $j=2, \ldots, k$. Then $N=N_{1} \times{ }_{1} N_{2} \times \cdots \times{ }_{1} N_{k}$ is the Euclidean $k$ space $\mathbb{E}^{k}$. Clearly, for a totally geodesic immersion of $N$ into $\mathbb{E}^{k+1}$, inequality (1.5) is false.

Example 3.2. Let $N_{1}=\{x \in \mathbf{R}: x>0\}$ and $N_{2}, \ldots, N_{k}$ be $k-1$ copies of $\mathbf{R}$. If we put $f_{2}=\cdots=f_{k}=x$, then each $f_{j}$ is 2 -imbalance for every 
$q_{j}>1$ and $N=N_{1} \times{ }_{x} N_{2} \times \cdots \times{ }_{x} N_{k}$ is an open subset of $\mathbb{E}^{k}$. Again, for a totally geodesic immersion of $N$ into $\mathbb{E}^{k+1}$, inequality (1.5) is false.

Remark 3.2. Theorems 1.1 and 1.2 are sharp in the sense that inequality (1.5) and (1.6) are false if either $f_{j}$ were constant or $f_{j}$ were 2 -imbalanced for every $q_{j}>1$ (For details, we refer to Remark 3.1, and Examples 3.1-3.2 above). Furthermore, Theorem 1.3 shows that inequality (1.5) (resp., (1.6)) is best possible for Theorem 1.1 (resp., for Theorem 1.2).

\section{Multiply Warped Product Manifolds into complex or quaternionic space forms}

A submanifold $N$ of a Kähler manifold $M$ is said to be totally real if the almost complex structure $J$ of $M$ carries each tangent space of $N$ into its corresponding normal space (cf. [8, 4]). Similarly, one has the notion of totally real submanifolds in quaternionic Kähler manifolds (cf. 7]).

B.-Y. Chen and F. Dillen proved

Theorem F [6] Let $\phi: N_{1} \times_{f_{2}} N_{2} \times \cdots \times \times_{f_{k}} N_{k} \rightarrow \tilde{M}^{m}(4 c)$ be a totally real isometric immersion of the multiply warped product $N=N_{1} \times{ }_{f_{2}} N_{2} \times \cdots \times{ }_{f_{k}}$ $N_{k}$ into a complex space form of constant holomorphic sectional curvature $4 c$ or in a quaternionic space form of constant quaternionic sectional curvature 4c. Then

$$
-\frac{n^{2}}{4} H^{2}-n_{1}\left(n-n_{1}\right) c \leq \sum_{j=2}^{k} n_{j} \frac{\Delta f_{j}}{f_{j}}, \quad n=\sum_{i=1}^{k} n_{i} .
$$

By applying the same techniques, i.e., Theorem 2.1 (Warping Functions Growth Estimates) and Proposition 2.1 (An Average Method in PDE), we also have the following results.

Theorem 4.1. If for each $j, 2 \leq j \leq k, f_{j}$ is nonconstant and 2-balanced for some $q_{j}>1$, then for any multiply warped product $N=N_{1} \times_{f_{2}} N_{2} \times$ $\cdots \times{ }_{f_{k}} N_{k}$ totally real isometrically immersed in a complex space form of constant holomorphic sectional curvature $4 c$ or in a quaternionic space form of constant quaternionic sectional curvature $4 c$, the mean curvature $H$ of $\phi$ satisfies

$$
H^{2}>\frac{-4 n_{1}\left(n-n_{1}\right) c}{n^{2}}
$$

at some points.

In particular, if each $f_{j}$ is nonconstant and in $L^{q_{j}}$ for some $q_{j}>1$, then (4.2) holds at some points.

As applications of Theorem 4.1, we have the following.

Corollary 4.1. If for each $j, 2 \leq j \leq k, f_{j}$ is nonconstant and 2-balanced for some $q_{j}>1$, then there does not exist a totally real minimal immersion 
of any multiply warped product $N=N_{1} \times f_{f_{2}} N_{2} \times \cdots \times{ }_{f_{k}} N_{k}$ into a complex space form of constant holomorphic sectional curvature $4 c \leq 0$ or into a quaternionic space form of constant quaternionic sectional curvature $4 c \leq 0$.

In particular, if each $f_{j}$ is nonconstant and in $L^{q_{j}}$ for some $q_{j}>1$, then there does not exist an isometric minimal immersion of $N=N_{1} \times f_{f_{2}} N_{2} \times$ $\cdots \times_{f_{k}} N_{k}$ into $\tilde{M}^{m}(0)$.

Another application of Theorem 4.1 is the following dichotomy.

Corollary 4.2. Suppose the squared mean curvature of an isometric immersion of a multiply warped product $N=N_{1} \times f_{2} N_{2} \times \cdots \times{f_{k}}_{k} N_{k}$ into a Riemannian manifold $R^{m}(c)$ of constant sectional curvature $c$ satisfies

$$
H^{2} \leq \frac{-4 n_{1}\left(n-n_{1}\right) c}{n^{2}}
$$

everywhere on $N$. Then there exists an integer $i, 2 \leq i \leq k$, such that the warping function $f_{i}$ is either a constant or for every $q_{i}>1, f_{i}$ has 2-imbalanced growth.

By applying the Growth Estimates in Theorem 2.1 and an Average Method in PDE in Proposition 2.1, we have the following.

Corollary 4.3. Suppose the squared mean curvature of a totally real isometric immersion $\phi$ of a multiply warped product $N=N_{1} \times{ }_{f_{2}} N_{2} \times \cdots \times{ }_{f_{k}} N_{k}$ into a complex space form of constant holomorphic sectional curvature $4 c$ or a quaternionic space form of constant quaternionic sectional curvature $4 c$ satisfies (4.3) everywhere on $N$. If for each $j, 2 \leq j \leq k, f_{j}$ is 2-balanced for some $q_{j}>1$, then

(1) Every warping function $f_{j}, 2 \leq j \leq k$, is constant.

(2) The isometric immersion $\phi$ is a minimal immersion into $\tilde{M}^{m}(0)$.

Corollary 4.4. Let each $f_{j}, 2 \leq j \leq k$, be 2-balanced for some $q_{j}>1$. Then

(1) Every multiply warped product $N=N_{1} \times{ }_{f_{2}} N_{2} \times \cdots \times{ }_{f_{k}} N_{k}$ does not admit an isometrically totally real minimal immersion into any complex space form of negative constant holomorphic sectional curvature $4 c$ or a quaternionic space form of negative constant quaternionic sectional curvature $4 c$.

(2) If $N_{1}$ is compact, then $N=N_{1} \times{ }_{f_{2}} N_{2} \times \cdots \times{ }_{f_{k}} N_{k}$ does not admit an isometrically totally real minimal immersion into $\tilde{M}^{m}(0)$.

We state a special case of Corollary 4.4 as follows.

Corollary 4.5. If each $f_{j}, 2 \leq j \leq k$, is in $L^{q_{j}}$ for some $q_{j}>1$, then we have:

(1) Every multiply warped product $N=N_{1} \times{ }_{f_{2}} N_{2} \times \cdots \times{ }_{f_{k}} N_{k}$ does not admit an isometrically totally real minimal immersion into a complex space form of negative constant holomorphic sectional curvature $4 \mathrm{c}$ 
or into a quaternionic space form of negative constant quaternionic sectional curvature $4 c$.

(2) If $N_{1}$ is compact, then $N_{1} \times{ }_{f_{2}} N_{2} \times \cdots \times{ }_{f_{k}} N_{k}$ does not admit an isometrically totally real minimal immersion into $\tilde{M}^{m}(0)$.

Since the proofs of these results can be done in the same way as in section 3 , we omit their proofs.

\section{Doubly Warped Products}

Doubly warped products are natural generalization of (ordinary) warped products.

Definition 5.1. A doubly warped product of Riemannian manifolds $\left(N_{1}, g_{1}\right)$ and $\left(N_{2}, g_{2}\right)$ is a product manifold ${ }_{f_{2}} N_{1} \times{ }_{f_{1}} N_{2}$ equipped with metric $g=$ $f_{2}^{2} g_{1} \oplus f_{1}^{2} g_{2}$, where $f_{1}: N_{1} \rightarrow \mathbb{R}^{+}$and $f_{2}: N_{2} \rightarrow \mathbb{R}^{+}$are positive-valued smooth functions.

As an extension of Theorem A from [9], A. Olteanu proved the following.

Theorem G [14] Let $\phi: f_{2} N_{1} \times{ }_{f_{1}} N_{2} \rightarrow M$ be an isometric immersion of a doubly warped product ${ }_{f_{2}} N_{1} \times{ }_{f_{1}} N_{2}$ into an arbitrary Riemannian manifold M. We have

$$
-\frac{\left(n_{1}+n_{2}\right)^{2}}{4} H^{2}-n_{1} n_{2} \max \tilde{K} \leq n_{2} \frac{\Delta_{1} f_{1}}{f_{1}}+n_{1} \frac{\Delta_{2} f_{2}}{f_{2}},
$$

where $n_{i}=\operatorname{dim} N_{i}$ and $\Delta_{i}$ is the Laplacian of $N_{i}$, for $i=1,2$.

The equality sign holds identically if and only if the following conditions hold:

i) $\phi$ is mixed totally geodesic such that trace $h_{1}=$ trace $h_{2}$;

ii) At each point $x=\left(x_{1}, x_{2}\right) \in N, \tilde{K}$ satisfies $\tilde{K}(u, v)=\max K(x)$ for each unit vector $u \in T_{x_{1}}^{1} N_{1}$ and every $v \in T_{x_{2}}^{1} N_{2}$.

Similarly, by applying the same techniques via Theorem 2.1 (Warping Functions Growth Estimates) and Proposition 2.1 (An Average Method in $\mathrm{PDE})$, we also have the following.

Theorem 5.1. If $f_{1}, f_{2}$ are nonconstant and 2-balanced for some $q_{1}, q_{2}>1$, then for any isometric immersion of a doubly warped product $\phi: f_{2} N_{1} \times{ }_{f_{1}} N_{2}$ into a Riemannian manifold $M$, the mean curvature $H$ of $\phi$ satisfies

$$
H^{2}>\frac{-4 n_{1} n_{2}}{\left(n_{1}+n_{2}\right)^{2}} \max \tilde{K}
$$

at some points.

In particular, if each $f_{j}$ is nonconstant and in $L^{q_{j}}$ for some $q_{j}>1$, then (5.2) holds at some points.

The proof of this theorem is similar to the proof of Theorem 2.1, However, because doubly warped products are somewhat different from ordinary warped products, we provide the proof of Theorem 5.1 as follows. 
Proof of Theorem 5.1. Suppose contrary to (5.2), i.e., there were an isometric immersion $\phi$ whose mean curvature $H$ satisfying

$$
H^{2} \leq \frac{-4 n_{1} n_{2}}{\left(n_{1}+n_{2}\right)^{2}} \max \tilde{K}
$$

everywhere on $N$, which gives

$$
0 \leq-\frac{\left(n_{1}+n_{2}\right)^{2}}{4} H^{2}-n_{1} n_{2} \max \tilde{K}
$$

On the other hand, Theorem G would imply

$$
-\frac{\left(n_{1}+n_{2}\right)^{2}}{4} H^{2}-n_{1} n_{2} \max \tilde{K} \leq n_{2} \frac{\Delta_{1} f_{1}}{f_{1}}+n_{1} \frac{\Delta_{2} f_{2}}{f_{2}} .
$$

After combining this with (5.3), we find

$$
n_{2} \frac{\Delta_{1} f_{1}}{f_{1}}+n_{1} \frac{\Delta_{2} f_{2}}{f_{2}} \geq-\frac{\left(n_{1}+n_{2}\right)^{2}}{4} H^{2}-n_{1} n_{2} \max \tilde{K} \geq 0 .
$$

After applying the Average Method in PDE stated in Proposition 2.1(1), (5.4) shows that some $f_{i}, i=1,2$, could be constant or $f_{i}$ would be 2imbalanced for every $q_{i}>1$. This contradicts the assumption that $f_{i}$ is nonconstant and 2-balanced for some $q_{i}>1$.

The last assertion follows from Definition 2.1, the first assertion of Theorems 5.1 and the fact that every $L^{q}$ function has 2-finite, 2-mild, 2-obtuse, 2-moderate, 2-small growth for the same $q$ (cf. [18, Proposition 2.3]).

In particular, if the ambient space $M$ is of constant sectional curvature $c \leq 0$, then Theorem 5.1 reduces to the following.

Theorem 5.2. If $f_{1}, f_{2}$ are nonconstant and 2-balanced for some $q_{1}, q_{2}>1$, then for any isometric immersion of a doubly warped product $\phi: f_{2} N_{1} \times_{f_{1}} N_{2}$ into a Riemannian m-manifold $R^{m}(c)$ of constant curvature $c \leq 0$, the mean curvature $H$ of $\phi$ satisfies

$$
H^{2}>\frac{-4 n_{1} n_{2}}{\left(n_{1}+n_{2}\right)^{2}} c
$$

at some points.

In particular, if each $f_{j}$ is nonconstant and in $L^{q_{j}}$ for some $q_{j}>1$, then (5.5) holds at some points.

Also, the following are easy consequences of Theorem 5.1.

Corollary 5.1. If for each $j(j=1,2), f_{j}$ is nonconstant and 2-balanced for some $q_{j}$, then there does not exist an isometric minimal immersion of any doubly warped product $\phi:{ }_{f_{2}} N_{1} \times{ }_{f_{1}} N_{2}$ into any negatively curved Riemannian manifold.

In particular, if each $f_{j}$ is nonconstant and in $L^{q_{j}}$ for some $q_{j}>1$, then there does not exist isometric minimal immersion of $N=N_{1} \times_{f_{2}} N_{2} \times \cdots \times{ }_{f_{k}}$ $N_{k}$ into a Euclidean space. 
As another easy applications of Theorem 5.1, we have the following dichotomy.

Corollary 5.2. Suppose the squared mean curvature of the isometric immersion of a doubly warped product $\phi: f_{2} \quad N_{1} \times{ }_{f_{1}} N_{2}$ into a Riemannian manifold satisfies

$$
H^{2} \leq \frac{-4 n_{1} n_{2}}{\left(n_{1}+n_{2}\right)^{2}} \max \tilde{K}
$$

everywhere on $N$. Then there exists an integer $i, 1 \leq i \leq 2$, such that the warping function $f_{i}$ is either a constant or for every $q_{i}>1, f_{i}$ has 2-imbalanced growth.

Analogously, by applying the growth estimates and the average method in PDE as before, we have the following Liouville property and a characterization result.

Corollary 5.3. Suppose the squared mean curvature of an isometric immersion $\phi$ of a doubly warped product $f_{2} N_{1} \times{ }_{f_{1}} N_{2}$ into a Riemannian manifold satisfies (5.3) on $N$. If for each $j(j=1,2), f_{j}$ is 2-balanced for some $q_{j}>1$, then we have:

(1) Every warping function $f_{j}, 1 \leq j \leq 2$, is constant.

(2) The isometric immersion $\phi$ is a minimal immersion into a Euclidean space.

Corollary 5.4. Let each $f_{j}, 1 \leq j \leq 2$ be 2-balanced for some $q_{j}>1$. Then we have:

(1) Every doubly warped product ${ }_{f_{2}} N_{1} \times_{f_{1}} N_{2}$ does not admit an isometric minimal immersion into any negatively curved Riemannian manifold.

(2) If $N_{2}$ is compact, then $f_{2} N_{1} \times{ }_{f_{1}} N_{2}$ does not admit an isometric minimal immersion into a Euclidean space.

We state a special case of Corollary 5.5 .

Corollary 5.5. If each $f_{j}(j=1,2)$ is in $L^{q_{j}}$ for some $q_{j}>1$, then we have:

(1) Every doubly warped product ${ }_{f_{2}} N_{1} \times_{f_{1}} N_{2}$ does not admit an isometric minimal immersion into any negatively curved Riemannian manifold.

(2) If $N_{2}$ is compact, then ${ }_{f_{2}} N_{1} \times_{f_{1}} N_{2}$ does not admit an isometric minimal immersion into a Euclidean space.

Since Corollaries 5.15 .5 can be in the same way as the proofs of Corollary 1.1, Theorem 1.3, Corollary 1.2 (1)\&(2), Corollary 1.3 and Corollary 1.4, we omit their proofs. 


\section{REFERENCES}

[1] Andreotti A. and Vesentini E., Carleman Estimates for the Laplace-Beltrami Equation on Complex Manifolds, Inst. Hautes Études Sci. Publ. Math. 25 (1965) 81-130.

[2] Chen B.-Y., Some Pinching and Classification Theorems for Minimal Submanifolds, Arch. Math. 60 (1993) 568-578.

[3] Chen B.-Y., On Isometric Minimal Immersion from Warped Products into Space Forms, Proc. Edinb. Math. Soc. 45 (2002) 579-587.

[4] Chen B.-Y., Pseudo-Riemannian Geometry, delta-invariants and Applications, World Scientific, Hackensack 2011.

[5] Chen B.-Y., Differential Geometry of Warped Product Manifolds and Submanifolds, World Scientific, Hackensack 2017.

[6] Chen B.-Y. and Dillen F., Optimal Inequalities for Multiply Warped Product Submanifolds, Int. Electron. J. Geom. 1(1) (2008), 1-11; Erratum, ibid. 4(1) (2011) p.138.

[7] Chen B.-Y. and Houh C. S., Totally Real Submanifolds of a Quaternion Projective Space, Ann. Mat. Pura Appl. 120 (1979) 185-199.

[8] Chen B.-Y. and Ogiue K., On Totally Real Submanifolds, Trans. Amer. Math. Soc. 193 (1974) 257-266.

[9] Chen B.-Y. and Wei S. W., Growth Estimates for Warping Functions and Their Geometric Applications, Glasg. Math. J. 51(3) (2009) 579-592.

[10] Howard R. and Wei S. W., On the Existence and Nonexistence of Stable Submanifolds and Currents in Positively Curved Manifolds and the Topology of Submanifolds in Euclidean Spaces, Geometry and Topology of Submanifolds and Currents, Contemp. Math. 646 (2015) 127-167.

[11] Karp L., Subharmonic Functions on Real and Complex Manifolds, Math. Z. 179 (1982) 535-554.

[12] Magid M., Isometric Immersions of Lorentz Space with Parallel Second Fundamental Forms, Tsukuba J. Math. 8(1) (1984) 31-54.

[13] Nölker S., Isometric Immersions of Warped Products, Differential Geom. Appl. 6(1) (1996) 1-30.

[14] Olteanu, A., A General Inequality for Doubly Warped Product Submanifolds, Math. J. Okayama Univ. 52 (2010) 133-142.

[15] Wei S. W., p-harmonic Geometry and Related Topics, Bull. Transilv. Univ. Brasov Ser. III 1(50) (2008) 415-453.

[16] Wei S. W., An Extrinsic Average Variational Method, Recent Developments in Geometry (Los Angeles, CA, 1987), Contemp. Math. 101 (1989), 55-78.

[17] Wei S. W., On Topological Vanishing Theorems and the Stability of Yang-Mills Fields, Indiana Univ. Math. J. 33(4) (1984) 511-529.

[18] Wei S. W., Li J.-F. and Wu L., Generalizations of the Uniformization Theorem and Bochner's Method in p-harmonic Geometry, Commun. Math. Anal. Conference 1 (2008) 46-68.

B.-Y. Chen, Department of Mathematics, Michigan State University, East Lansing, Michigan 48824-1027, U.S.A.

E-mail address: chenb@msu.edu

S. W. Wei, Department of Mathematics, University of Oklahoma, Norman, Oklahoma 73019-0315, U.S.A.

E-mail address: wwei@ou.edu 